\title{
An Explicit Formula of Hitting Times for Random Walks on Graphs
}

\author{
HaO Xu and Shing-Tung Yau
}

\begin{abstract}
We prove an explicit formula of hitting times in terms of enumerations of spanning trees for random walks on general connected graphs. We apply the formula to improve Lawler's bound of hitting times for general graphs and derive closed formulas of hitting times for some special graphs.
\end{abstract}

Keywords: Random walk, hitting time, spanning tree.

\section{Introduction}

Unless otherwise specified, throughout the paper, we assume $G=(V, E)$ to be an undirected graph with $n=|V|$ vertices and without multi-edges or loops. The volume of $G$ is $\operatorname{vol}(G)=\sum_{v \in V} d_{v}$, where $d_{v}$ is the degree of $v$. Let $\tau(G)$ be the number of spanning trees of $G$. The Laplacian of $G$ is the matrix $L=D-A$, where $D$ is the diagonal matrix whose entries are the degree of the vertices and $A$ is the adjacency matrix of $G$. For $x, y \in V$, $x \sim y$ denotes that they are adjacent vertices.

When $G$ is connected, the eigenvalues of Chung's normalized Laplacian $\mathcal{L}=D^{-1 / 2} L D^{-1 / 2}$ can be labeled by $0=\lambda_{1}<\lambda_{2} \leq \lambda_{3} \leq \cdots \leq \lambda_{n}$ with the corresponding orthonormal basis of eigenvectors $v_{1}, v_{2}, \ldots, v_{n}$. Let $v_{i}=$ $\left(v_{i 1}, \ldots, v_{i n}\right)^{t}$. Obviously $v_{1}(x)=\sqrt{d_{x} / \operatorname{vol}(G)}, \forall x \in V$.

A random walk on $G$ is a time-reversible finite Markov chain that begins at some vertex, and at each step moves to a neighbor of the present vertex $x$ with probability $1 / d_{x}$. The hitting time $H(x, y)$ is the expected number of steps to reach vertex $y$, when started from vertex $x$. An excellent comprehensive survey of random walks on graphs can be found in [13.

Received September 14, 2013.

MSC(2010) 05C81 (05C50 60G50). 
Chung and Yau [7] (see also [6]) proved an explicit formula of $H(x, y)$ in terms of the discrete Green function

$$
H(x, y)=\operatorname{vol}(G)\left(\frac{\mathscr{G}(y, y)}{d_{y}}-\frac{\mathscr{G}(x, y)}{\sqrt{d_{x} d_{y}}}\right) .
$$

The discrete Green function $\mathscr{G}$ is uniquely defined by the equations

$$
\mathscr{G} \mathcal{L}=\mathcal{L} \mathscr{G}=I-P_{0}, \quad \mathscr{G} P_{0}=0, \quad P_{0}=v_{1} v_{1}^{t} .
$$

Chung-Yau's formula (1] is the starting point of our work [16], which is continued here.

Lovász proved a remarkable formula [13, Thm. 3.1] connecting hitting times to spectra of $\mathcal{L}$.

$$
H(x, y)=\operatorname{vol}(G) \sum_{k=2}^{n} \frac{1}{\lambda_{k}}\left(\frac{v_{k y}^{2}}{d_{y}}-\frac{v_{k x} v_{k y}}{\sqrt{d_{x} d_{y}}}\right) .
$$

Note that the original formula of Lovász was formulated using eigenvalues and eigenvectors of the matrix $I-\mathcal{L}$. By definition (see Eq. (10) of [7]), $\mathscr{G}(x, y)=\sum_{i=2}^{n} \frac{1}{\lambda_{k}} v_{k x} v_{k y}$, so (2) follows from (1).

Consider the graph $G$ as an electrical network, where each edge has unit resistance. Tetali's electrical formula [15] provides a powerful approach to the computation of hitting times.

$$
H(x, y)=\frac{1}{2} \sum_{z \in V(G)} d_{z}\left(R_{x y}+R_{y z}-R_{x z}\right),
$$

where $R_{x y}$ is the effective resistance between $x$ and $y$.

The paper is organized as follows: In $\$ 2$, we briefly review our previous work and prove an explicit formula of $H(x, y)$ in Theorem 2.7 together with some interesting applications. In $\$ 3$, we present a proof of Tetali's electrical formula. In $\$ 4$, we apply our formula to recover some identities of hitting times of random walks on lollipop graphs and unicycle graphs.

Acknowledgements We thank Stephan Wagner for helpful comments on an earlier version of this paper. We also thank a referee for providing an alternative proof of Corollary 2.12 (see Remark 2.14). 


\section{Explicit formulas of Hitting times}

A vertex-weighted graph is a graph $G$ together with a weight function $w$ : $V(G) \rightarrow \mathbb{R}$. In our case, $w_{x}$ at $x \in V(G)$ will usually be the degree of $x$ in some ambient graph of $G$. So we may assume $d_{x} \leq w_{x} \in \mathbb{Z}$. Denote by $d_{G}$ the weight function that takes $d_{x}$ for each $x \in V(G)$.

In [16, we defined two invariants $R(G, w)$ and $Z(G, w)$ for a vertexweighted graph $(G, w)$. For the empty graph $\emptyset$, we define $R(\emptyset, w)=1$ and $Z(\emptyset, w)=0$. For any given vertex $x \in V(G)$, they satisfy the recursive formulas

$$
\begin{aligned}
& R(G, w)=w_{x} R(G-\{x\}, w)-\sum_{\substack{y \in V(G) \\
y \sim x}} \sum_{P \in \mathscr{P}_{G}(x, y)} R(G-\{P\}, w), \\
& Z(G, w)=w_{x} Z(G-\{x\}, w)-\sum_{\substack{y \in V(G) \\
y \sim x}} \sum_{\substack{\left.y \in \mathscr{P}_{G} \\
y, y\right)}} Z(G-\{P\}, w) \\
& +w_{x}^{2} R(G-\{x\}, w)+\sum_{\substack{u, v \in V(G) \\
u \neq v}} \sum_{\substack{P_{1} \in \mathscr{P}_{G}(x, u) \\
P_{2} \in \mathscr{P}_{G}(x, v) \\
P_{1} \cap P_{2}=x}} w_{u} w_{v} R\left(G-\left\{P_{1}, P_{2}\right\}, w\right) .
\end{aligned}
$$

where $\mathscr{P}_{G}(x, y)$ is the set of all simple undirected paths (with no repeated vertices) connecting $x$ and $y$ in $G$. By convention $\mathscr{P}_{G}(x, x)$ consists of the trivial path $\{x\}$ only. Here $(G-\{P\}, w)$ means the restriction of $w$ to the subgraph $G-\{P\}$. Note that (4) and (5) uniquely determine these invariants.

The invariants $R(G, w)$ and $Z(G, w)$ enjoy the following nice properties.

Lemma 2.1 ([16]). If $G$ has $k$ connected components $G_{1}, \ldots, G_{k}$, then

$$
R(G, w)=\prod_{i=1}^{k} R\left(G_{i}, w\right), \quad Z(G, w)=\sum_{i=1}^{k} Z\left(G_{i}, w\right) \prod_{\substack{j=1 \\ j \neq i}}^{k} R\left(G_{j}, w\right)
$$

Lemma 2.2 ([16]). We have

$$
Z(G, w)=\sum_{x, y \in V(G)} \sum_{P \in \mathscr{P}_{G}(x, y)} w_{x} w_{y} R(G-\{P\}, w) .
$$


Lemma $2.3([\mathbf{1 6}])$. Let $G$ be a connected graph. Then we have $R\left(G, d_{G}\right)=$ 0 and $Z\left(G, d_{G}\right)=\operatorname{vol}(G)^{2} \tau(G)$. For any $x, y \in V(G)$, we have

$$
R\left(G-\{x\}, d_{G}\right)=\sum_{P \in \mathscr{P}_{G}(x, y)} R\left(G-\{P\}, d_{G}\right)=\tau(G) .
$$

Remark 2.4. Recall a well-known result from linear algebra: Let $G$ be a connected graph with possibly multi-edges but no loops. Let $L$ be its Laplacian matrix and $L^{\prime}$ the matrix obtained by deleting the first row and column from $L$. Then

$$
\tau(G)=\operatorname{det}\left(L^{\prime}\right)
$$

Given a vertex-weighted graph $(G, w)$, define the completion graph $\bar{G}$ of $G$ to be a multi-graph with $V(\bar{G})=V(G) \cup\{\bullet\}$ and $E(\bar{G})$ consists of $E(G)$ plus $w_{v}-d_{v}$ newly added edges between $\bullet$ and $v \in V(G)$ for each $v \in V(G)$. It is not difficult to see that $R(G, w)=\tau(\bar{G})$. See the proof of [16, Lem. 2.13] for details.

The main result of [16] is an explicit formula of hitting times in terms of the invariants $R(G, w)$ and $Z(G, w)$.

Theorem 2.5 ([16]). Let $G$ be a connected graph and $x, y \in V(G)$. Then

$$
\begin{aligned}
H(x, y)=\frac{1}{\operatorname{vol}(G) \tau(G)} & \left(Z\left(G-\{y\}, d_{G}\right)-\sum_{P \in \mathscr{P}_{G}(x, y)} Z\left(G-\{P\}, d_{G}\right)\right. \\
& \left.+\sum_{\substack{u, v \in V(G) \\
u \neq v}} \sum_{\substack{P_{1} \in \mathscr{P}_{G}(x, u) \\
P_{2} \in \mathscr{P}_{G}(y, v) \\
P_{1} \cap P_{2}=\emptyset}} d_{u} d_{v} R\left(G-\left\{P_{1}, P_{2}\right\}, d_{G}\right)\right) .
\end{aligned}
$$

Remark 2.6. According to [10, a graph $G$ is called reversible if $H(x, y)=$ $H(y, x)$ holds for any $x, y \in V(G)$. It is not difficult to see that $(9)$ implies that $G$ is reversible if and only if $Z\left(G-\{x\}, d_{G}\right)$ is independent of the vertex $x$. An immediate corollary is that vertex-transitive graphs are reversible. See [13, Cor. 2.6] for an alternative proof of this assertion. It is interesting to compare with the result in [15. Cor. 4] (also cf. [11]) that $G$ is reversible if and only if $\sum_{u \in V(G)} d_{u} R_{v u}$ is independent of the vertex $v$, where $R_{v u}$ is the effective resistance between $v$ and $u$. In [4], we will apply our work to study the interesting problems on reversible graphs posed by Georgakopoulos [10]. 
Theorem 2.7. Let $G$ be a connected graph and $x, y \in V(G)$. Then

$$
H(x, y)=\frac{1}{\tau(G)} \sum_{u \in V(G)} d_{u} \sum_{\substack{P \in \mathscr{P}_{G}(x, u) \\ y \notin P}} R\left(G-\{P, y\}, d_{G}\right) .
$$

In fact, $R\left(G-\{P, y\}, d_{G}\right)=\tau(G /\{P, y\})$.

Proof. By Lemma 2.2 and Remark 2.4 the formula $(9)$ is equivalent to

$$
\begin{aligned}
H(x, y)= & \frac{1}{\operatorname{vol}(G) \tau(G)} \sum_{u, v \in V(G)} d_{u} d_{v}\left(\sum_{\substack{P \in \mathscr{P}_{G}(u, v) \\
y \notin P}} \tau(G /\{P, y\})\right. \\
& \left.-\sum_{\substack{P_{1} \in \mathscr{P}_{G}(x, y) \\
P_{2} \in \mathscr{P}_{G}(x, v) \\
P_{1} \cap P_{2}=\emptyset}} \tau\left(G /\left\{P_{1}, P_{2}\right\}\right)+\sum_{\substack{P_{1} \in \mathscr{P}_{G}(x, u) \\
P_{2} \in \mathscr{P}_{G}(y, v) \\
P_{1} \cap P_{2}=\emptyset}} \tau\left(G /\left\{P_{1}, P_{2}\right\}\right)\right),
\end{aligned}
$$

where $G /\{P, y\}$ and $G /\left\{P_{1}, P_{2}\right\}$ denote (multi-)graphs obtained from $G$ by contracting $\{P, y\}$ and $\left\{P_{1}, P_{2}\right\}$ to a vertex respectively.

Denote by $F(x, y, u, v)$ the bracket term of (11). We will show that for any fixed vertices $x, y, u, F(x, y, u, v)$ is independent of $v$. This is obvious when $x=y$ or $u=y$, which forces $F(x, y, u, v)=0, \forall v \in V(G)$. When $v=x$ or $y$, we have

$$
F(x, y, u, x)=F(x, y, u, y)=\sum_{\substack{P \in \mathscr{P}_{G}(x, u) \\ y \notin P}} \tau(G /\{P, y\}) .
$$

Assume $v \neq x, y$, we modify $G$ by adding an edge $u y$ if $u, y$ are not adjacent, namely we define a simple graph $G^{\prime}$ by

$$
G^{\prime}= \begin{cases}G & \text { if } u \sim y, \\ G \cup\{u y\} & \text { otherwise. }\end{cases}
$$

Denote by $\Omega\left(G^{\prime}\right)$ the set of spanning trees of $G^{\prime}$. Each of the three summands in $F(x, y, u, v)$ counts a subset of $\Omega\left(G^{\prime}\right)$. They are respectively equal to

$\#\left\{T \in \Omega\left(G^{\prime}\right) \mid T\right.$ contains $u y$ and a path from $u$ to $v$ not containing $\left.y\right\}$, $\#\left\{T \in \Omega\left(G^{\prime}\right) \mid T\right.$ contains $u y$, a path $P_{1}$ from $x$ to $y$ and a path $P_{2}$ from $u$ to $v$ such that $\left.P_{1} \cap P_{2}=\emptyset\right\}$, 
$\#\left\{T \in \Omega\left(G^{\prime}\right) \mid T\right.$ contains $u y$, a path $P_{1}$ from $u$ to $x$ and a path $P_{2}$ from $v$ to $y$ such that $P_{1} \cap P_{2}=\emptyset$.

It is not difficult to see that

$F(x, y, u, v)=114-15)+16$

$=\#\left\{T \in \Omega\left(G^{\prime}\right) \mid T\right.$ contains $u y$ and a path from $u$ to $x$ not containing $\left.y\right\}$

$=F(x, y, u, x)$,

which proves that $F(x, y, u, v)$ is independent of $v \in V(G)$. The last equation used (12). Since $\tau(G /\{P, y\})=R\left(G-\{P, y\}, d_{G}\right)$, we get (10) immediately from (11).

As an application of the above theorem, we give a simple proof of the well-known inequality $H(x, y) \leq O\left(n^{3}\right)$, where $n=|V(G)|$.

Corollary 2.8. Let $G$ be a connected graph with $n$ vertices and $x, y \in V(G)$. Then

$$
H(x, y) \leq(n-1)^{3} .
$$

If in addition $d_{x} \leq k, \forall x \in V(G)$, then

$$
H(x, y) \leq k(n-1)^{2} .
$$

Proof. Fix $x, y, u \in V(G)$ with $y \neq u$. Given a spanning tree $T \in \Omega(G)$ and an edge $e \in E(T)$, denote by $T(e)$ a subgraph of $G^{\prime}$ (defined in (13)) obtained from $T$ by removing $e$ and adding an edge $u y$ if $u y \notin E(T)$, namely

$$
T(e)= \begin{cases}T & \text { if } u y \in T, \\ \{T-e\} \cup\{u y\} & \text { if } u y \notin T .\end{cases}
$$

Define a subset $S$ of $\Omega(G) \times E(G)$ by

$$
S=\left\{(T, e) \mid T \in \Omega(G), e \in E(T), T(e) \in \Omega\left(G^{\prime}\right)\right\}
$$

and $S^{\prime}=\left\{T \in \Omega\left(G^{\prime}\right) \mid T\right.$ contains $\left.u y\right\}$. Then the map $(T, e) \rightarrow T(e)$ is a surjective map from $S$ to $S^{\prime}$. Therefore we have

$$
\sum_{\substack{P \in \mathscr{P}_{G}(x, u) \\ y \notin P}} \tau(G /\{P, y\})
$$


$=\#\left\{T \in \Omega\left(G^{\prime}\right) \mid T\right.$ contains $u y$ and a path from $u$ to $x$ not containing $\left.y\right\}$ $\leq\left|S^{\prime}\right| \leq|S| \leq(n-1) \tau(G)$.

Let $d_{\max }=\max \left\{d_{v} \mid v \in V(G)\right\}$. Then from (10), we have

$$
H(x, y) \leq d_{\max }(n-1)^{2},
$$

which implies (17) and (18).

Remark 2.9. An $O\left(n^{3}\right)$ upper bound for hitting and cover times was first proved by Aleliunas et al. [1. Inequalities (17) and (18) with slightly weaker bounds $n(n-1)^{2}$ and $k n(n-1)$ respectively were obtained by Lawler [12. A sharp bound of $H(x, y)$ with leading term $(4 / 27) n^{3}$ was obtained by Brightwell and Winkler [2], who also showed that lollipop graphs maximize $H(x, y)$.

Corollary 2.10. Let $G$ be a connected graph with $m$ edges and $x y \in E(G)$. Then

$$
H(x, y) \leq 2 m-d_{y}
$$

Proof. For any $u \in V(G)$ with $u \neq y$, it is not difficult to see that

$$
\begin{aligned}
& \sum_{\substack{P \in \mathscr{P}_{G}(x, u) \\
y \notin P}} \tau(G /\{P, y\}) \\
&=\#\{T \in \Omega(G) \mid T \text { contains } x y \text { and a path from } u \text { to } x \text { not containing } y\} \\
& \leq \tau(G) .
\end{aligned}
$$

Thus (10) implies that

$$
H(x, y) \leq \frac{1}{\tau(G)} \sum_{\substack{u \in V(G) \\ u \neq y}} d_{u} \tau(G)=2 m-d_{y}
$$

as claimed.

Remark 2.11. It is well-known (cf. [13, p.8]) that the commute time $\kappa(x, y):=H(x, y)+H(y, x) \leq 2 m$ whenever $x y \in E(G)$. The inequality (19) is sharp for the path graph, where $x$ and $y$ are respectively the next-to-right 
endpoint and the right endpoint. It can also be derived from the well-know identity

$$
1+\frac{1}{d_{y}} \sum_{\substack{x \in V(G) \\ x \sim y}} H(x, y)=\frac{2 m}{d_{y}} .
$$

Let $\mathscr{S}=\{u \in V(G) \mid$ There is a path from $x$ to $u$ not passing through $y\}$. If $x y \in E(G)$ is a cut edge of $G$, then it was proved in [1, 9] that $\kappa(x, y)=2 m$, or equivalently $H(x, y)=2\left|E\left(G^{\prime}\right)\right|-1$, where $G^{\prime}$ is the subgraph obtained by removing from $G$ all vertices not in $\{\mathscr{S} \cup y\}$. The latter equality can also be proved easily using (10). First note that $H(x, y)$ is the same for random walks on either $G$ and $G^{\prime}$. Moreover, for each spanning tree $T$ of $G^{\prime}$ and $u \in \mathscr{S}$, there exists a path from $x$ to $u$. Therefore,

$$
H(x, y)=\frac{1}{\tau\left(G^{\prime}\right)} \sum_{u \in \mathscr{S}} d_{u} \tau\left(G^{\prime}\right)=2\left|E\left(G^{\prime}\right)\right|-1,
$$

where the last equation follows from the fact that the degree of $y$ in $G^{\prime}$ is equal to 1 .

Corollary 2.12. Let $G$ be a connected graph on $n$ vertices. If there is a vertex $y$ with degree $n-1$, then for any $x \in V(G)$ we have

$$
H(x, y) \leq \max \left\{d_{u} \mid u \in \mathscr{S}\right\},
$$

where $\mathscr{S}=\{u \in V(G) \mid$ There is a path from $x$ to $u$ not passing through $y\}$.

Proof. Fix any $x \in V(G)$ with $x \neq y$, we define $\Omega_{x y}=\{T \in \Omega(G) \mid x y \in T\}$ and

$V_{T}=\{u \in V(G) \mid T$ contains a path from $x$ to $u$ not passing through $y\}$.

Let $S=\left\{(T, u) \mid T \in \Omega_{x y}, u \in V_{T}\right\}$. Define a map $f: S \rightarrow \Omega(G)$ by

$$
f(T, u)= \begin{cases}T & \text { if } u=x, \\ \{T-x y\} \cup\{u y\} & \text { if } u \neq x,\end{cases}
$$

where we used the fact that $d_{y}=n-1$. It is not difficult to see that $f$ is injective. Thus we have

$$
\sum_{u \in V(G)} \sum_{\substack{P \in \mathscr{P}_{G}(x, u) \\ y \notin P}} \tau(G /\{P, y\})=|S| \leq \tau(G) .
$$

Therefore (10) implies 20). 
Remark 2.13. Without loss of generality, we may assume $\mathscr{S}=V(G)-$ $\{y\}$ in the above corollary. Eq. 20 refines the result of Palacios 14, Thm. 3.1 , who proved $H(x, y) \leq n-1$ under the same condition of the above corollary by using inequalities between matrix norms.

Remark 2.14. A simple probabilistic proof of Corollary 2.12 was provided by a referee: Since the probability of moving to $\mathrm{y}$ is at least $p=1 / \max \left\{d_{u} \mid\right.$ $u \in \mathscr{S}\}$ in every step, the expected hitting time is at most the expected value of a geometric random variable with parameter $p$, which is $1 / p$.

Remark 2.15. Spanning trees have been extensively used to estimate hitting times [1, 8, 9], in combination with the theory of electric networks. It shall be interesting to see how to apply 10 to recover their estimates of hitting times. Explicit formulas of hitting times valid on general graphs are very rare. As shown in [16, §4], Eq. (9) is very useful in studying hitting times on general graphs. We will show in $\$ 4$ that Eq. (10) is very efficient in getting closed formulas for hitting times on graphs with few cycles.

\section{Random walks and electric networks}

There has been a large amount of work on connections between electrical networks and random walks on graphs. Chandra et al. 3. proved that the commute time $\kappa(x, y)$ can be expressed in terms of the effective resistance $\kappa(x, y)=\operatorname{vol}(G) R_{x y}$. Tetali's electrical formula [15], expressing $H(x, y)$ in terms of the effective resistance, was originally proved by using the reciprocity theorem of electrical networks. It was used to prove, among others, closed formulas of hitting times for trees and unicycle graphs [5]. As an illustration of the effectiveness of (9), we use it to prove Tetali's formula.

Theorem 3.1 (Tetali [15]). On a connected graph $G$,

$$
H(i, j)=\frac{1}{2}\left(\kappa(i, j)+\sum_{q \in V(G)} \frac{d_{q}}{\operatorname{vol}(G)}[\kappa(q, j)-\kappa(q, i)]\right) .
$$

Proof. By (9),

$$
\operatorname{vol}(G)[2 H(i, j)-\kappa(j, i)]=\operatorname{vol}(G)[H(i, j)-H(j, i)]
$$




$$
=\frac{1}{\tau(G)}\left(Z\left(G-\{j\}, d_{G}\right)-Z\left(G-\{i\}, d_{G}\right)\right) .
$$

and by (9) and (7),

$$
\begin{aligned}
& \sum_{q \in V(G)} d_{q}[H(q, j)+H(j, q)] \\
& =\frac{1}{\operatorname{vol}(G) \tau(G)}\left(\sum_{q \in V(G)} d_{q}\left[Z\left(G-\{q\}, d_{G}\right)+Z\left(G-\{j\}, d_{G}\right)\right]\right. \\
& -2 \sum_{q \in V(G)} d_{q} \sum_{P \in \mathscr{P}_{G}(q, j)} Z\left(G-\{P\}, d_{G}\right)
\end{aligned}
$$

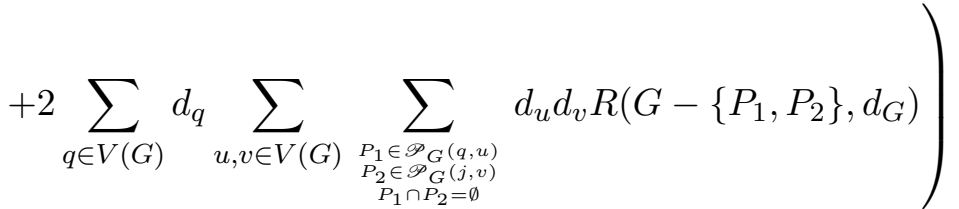

$$
\begin{aligned}
& =\frac{1}{\operatorname{vol}(G) \tau(G)} \sum_{q \in V(G)} d_{q}\left[Z\left(G-\{q\}, d_{G}\right)+Z\left(G-\{j\}, d_{G}\right)\right]
\end{aligned}
$$

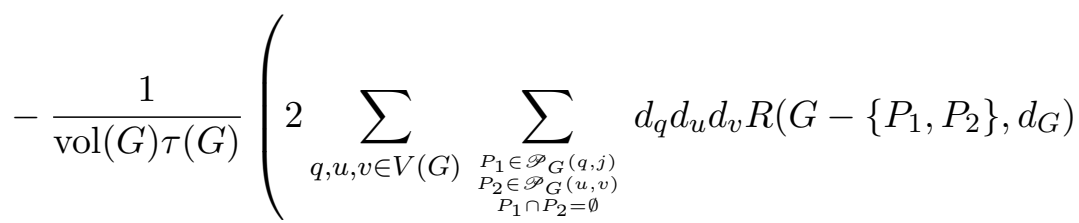

$$
\begin{aligned}
& \left.-2 \sum_{q, u, v \in V(G)} \sum_{\substack{P_{1} \in \mathscr{P}_{G}(q, u) \\
P_{2} \in \mathscr{P}_{G}(j, v) \\
P_{1} \cap P_{2}=\emptyset}} d_{q} d_{u} d_{v} R\left(G-\left\{P_{1}, P_{2}\right\}, d_{G}\right)\right) \\
& =\frac{1}{\operatorname{vol}(G) \tau(G)} \sum_{q \in V(G)} d_{q}\left[Z\left(G-\{q\}, d_{G}\right)+Z\left(G-\{j\}, d_{G}\right)\right] .
\end{aligned}
$$

The vanishing of the bracket term in the last equation can be seen by switching $q$ and $v$. By (23), we get

$$
\begin{gathered}
\sum_{q \in V(G)} d_{q}[\kappa(q, j)-\kappa(q, i)]=\sum_{q \in V(G)} d_{q}[H(q, j)+H(j, q)-H(q, i)-H(i, q)] \\
=\frac{1}{\operatorname{vol}(G) \tau(G)} \sum_{q \in V(G)} d_{q}\left(Z\left(G-\{j\}, d_{G}\right)-Z\left(G-\{i\}, d_{G}\right)\right)
\end{gathered}
$$


which, together with (22), implies Tetali's formula (21).

Tetali's formula (21) also gives an expression of hitting times in terms of numbers of spanning trees via the following equation (cf. [13])

$$
\kappa(x, y)=\operatorname{vol}(G) \frac{\tau\left(G^{\prime}\right)}{\tau(G)},
$$

where $x \neq y \in V(G)$ and $G^{\prime}$ is the graph obtained from $G$ by identifying $x$ and $y$. In contrast to Tetali's formula, the formula (10) does not have negative terms and thus is more efficient for bounding hitting times.

\section{Two examples}

The weight function $w$ may be written as a sequence $\left[w_{1}, \ldots, w_{n}\right]$ with $n=$ $|V(G)|$ once we specify a natural labeling of $V(G)$. The following lemmas will be used in Example 4.3 .

Lemma $4.1([\mathbf{1 6}, \S 3])$. Let $P_{n}$ and $K_{n}$ be the path and the complete graph on $n$ vertices respectively. Then

$$
\begin{aligned}
R\left(P_{n},\left[2^{n}\right]\right) & =n+1, \\
k_{n, m}:=R\left(K_{n},\left[m^{n}\right]\right) & =(m-n+1)(m+1)^{n-1} .
\end{aligned}
$$

Lemma 4.2. Let $L_{m, n}$ be a lollipop graph obtained by attaching a path $P_{n}$ to $K_{m}$.

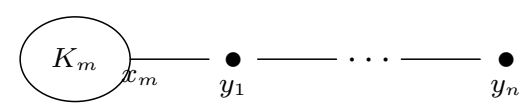

More precisely $V(G)=\left\{x_{1}, \ldots, x_{m}, y_{1}, \ldots, y_{n}\right\}$ and $\left(x_{i}, x_{j}\right) \in E(G)$ for $1 \leq$ $i<j \leq m ;\left(x_{m}, y_{1}\right) \in E(G) ;\left(y_{i}, y_{i}+1\right) \in E(G)$ for $1 \leq i<n$. Define a weight function $D_{k}$ on $L_{m, n}$ by

$$
D_{k}(v)= \begin{cases}d_{v}+k & \text { if } v=x_{j}, 1 \leq j \leq m, \\ 2 & \text { if } v=y_{j}, 1 \leq j \leq n .\end{cases}
$$

Then $r_{m, n, k}:=R\left(L_{m, n}, D_{k}\right)$ is equal to

$r_{m, n, k}=((m+k)(n+1)-n)(k+1)(m+k)^{m-2}-(m-1)(m+k)^{m-2}(n+1)$.

Proof. Taking $x=x_{m}$ in (4), we have 


$$
\begin{gathered}
R\left(G_{m, n}, D_{k}\right)=(m+k) R\left(K_{m-1},\left[(m-1+k)^{m-1}\right]\right) \cdot R\left(P_{n},\left[2^{n}\right]\right) \\
-R\left(K_{m-1},\left[(m-1+k)^{m-1}\right] \cdot R\left(P_{n-1},\left[2^{n-1}\right]\right)\right. \\
-(m-1) \sum_{i=0}^{m-2}\left(\begin{array}{c}
m-2 \\
i
\end{array}\right) i ! R\left(K_{m-2-i},(m-1+k)^{m-2-i}\right) R\left(P_{n},\left[2^{n}\right]\right) \\
=((m+k)(n+1)-n)(k+1)(m+k)^{m-2}-(m-1)(m+k)^{m-2}(n+1),
\end{gathered}
$$

as claimed.

Example 4.3. Let $G$ be a lollipop graph $L_{N, N}$ with $N \geq 2$.

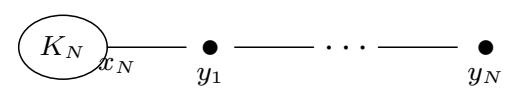

By 10$)$, we have

$$
\begin{aligned}
& H\left(x_{1}, y_{N}\right)=\frac{1}{N^{N-2}}\left((N-1) r_{N-1, N-1,1}\right. \\
& +(N-1)(N-2) \sum_{i=0}^{N-3}\left(\begin{array}{c}
N-3 \\
i
\end{array}\right) i ! r_{N-2-i, N-1, i+2} \\
& +(N-1)(N-2) \sum_{i=0}^{N-3}\left(\begin{array}{c}
N-3 \\
i
\end{array}\right)(i+1) \text { ! } \\
& \times R\left(K_{N-(i+3)},\left[(N-1)^{N-(i+3)}\right]\right) R\left(P_{N-1},\left[2^{N-1}\right]\right) \\
& +N \sum_{i=0}^{N-2}\left(\begin{array}{c}
N-2 \\
i
\end{array}\right) i ! R\left(K_{N-(i+2)},\left[(N-1)^{N-(i+2)}\right]\right) R\left(P_{N-1},\left[2^{N-1}\right]\right) \\
& +2 \sum_{i=0}^{N-2}\left(\begin{array}{c}
N-2 \\
i
\end{array}\right) i ! R\left(K_{N-(i+2)},\left[(N-1)^{N-(i+2)}\right]\right) \sum_{j=1}^{N-1} R\left(P_{N-1-j},\left[2^{N-1-j}\right]\right) \text {. }
\end{aligned}
$$

The five terms in the bracket respectively correspond to (i) $u=x_{1}$; (ii) $u=x_{j}, 2 \leq j \leq N-1$ and $x_{N} \notin P \in \mathscr{P}_{G}\left(x_{1}, u\right)$; (iii) $u=x_{j}, 2 \leq j \leq N-1$ and $x_{N} \in P \in \mathscr{P}_{G}\left(x_{1}, u\right)$; (iv) $u=x_{N}$; (v) $u=y_{j}, 1 \leq j \leq N-1$.

By Lemmas 4.1 and 4.2 , it is not difficult to get

(28) $H\left(x_{1}, y_{N}\right)=\frac{1}{N^{N-2}}\left((N-1) r_{N-1, N-1,1}\right.$

$+(N-1)(N-2) \sum_{i=0}^{N-3} \frac{N-3}{(N-3-i) !}\left[r_{N-2-i, N-1, i+2}+(i+1) k_{N-(i+3),(N-1)} \cdot N\right]$ 


$$
\begin{array}{r}
\left.+\sum_{i=0}^{N-2} \frac{(N-2) !}{(N-2-i) !}\left[k_{N-(i+2),(N-1)} \cdot N^{2}+2 k_{N-(i+2), N-1} \sum_{j=1}^{N-1}(N-j)\right]\right) \\
=N^{3}+N-1 .
\end{array}
$$

The above example was discussed in [12, 14] by different approaches. By using Tetali's electrical formula, Chen and Zhang [5] obtained an explicit formula for hitting times of random walks on unicycle graphs. In the next example, we apply Theorem 2.7 to give a more direct derivation of ChenZhang's formula.

Example 4.4. We follow the notations of [5]. Let $G$ be a connected unicyclic graph with a unique cycle $C$ of length $l$. Let $V(C)=\{1,2, \ldots, l\}$ and $T_{i}, 1 \leq i \leq l$ the tree component of $G \backslash E(C)$ containing $i$. Denote $m_{i}=$ $\left|E\left(T_{i}\right)\right|$. Given $i, j, k \in V(C)$, denote by $P_{i j k}$ the path from $i$ to $k$ containing $j$. Let $m_{i j}$ and $m_{j k}$ be the lengths of subpaths of $P_{i j k}$ from $i$ to $j$ and $j$ to $k$ respectively.

First we assume that there are two distinct vertices $i, j \in V(C)$ such that $a \in V\left(T_{i}\right), b \in V\left(T_{j}\right)$. Let $G_{0}$ be the subgraph of $G$ induced by $V\left(P_{a i}\right) \cup$ $V\left(P_{j b}\right) \cup V(C)$ and $m_{v}$ the number of edges in the component of $G \backslash E\left(G_{0}\right)$ containing $v$. Define a map $f: V(G) \rightarrow V\left(G_{0}\right)$ by setting $f(u)=v$ if $u \in$ $V(G)$ belongs to the component of $G \backslash E\left(G_{0}\right)$ containing $v \in V\left(G_{0}\right)$.

In (10), we deal with the three cases $u \in f^{-1}\left(P_{a i}\right), u \in f^{-1}\left(P_{j b}\right)$ and $u \in f^{-1}(V(C) \backslash\{i, j\})$ and get

$$
\begin{gathered}
H(a, b)=\frac{1}{l} \sum_{u \in V(G)} d_{u} \sum_{\substack{P \in \mathscr{P}_{G}(a, u) \\
y \notin P}} \tau(G /\{P, b\}) \\
=\sum_{v \in P_{a i}} 2 m_{v}\left(d(v, i)+d(j, b)+\frac{d(i, j)(l-d(i, j))}{l}\right)+\sum_{v \in P_{j b}} 2 m_{v} d(v, b) \\
\quad+\sum_{k \in V(C) \backslash\{i, j\}}\left(2 m_{k}+2\right)\left(d(j, b)+\frac{m_{i j} m_{j k}}{l}\right) \\
=2 \sum_{v \in P_{a i}} m_{v}\left(d(v, i)+d(j, b)+\frac{d(i, j)(l-d(i, j))}{l}\right)+2 \sum_{v \in P_{j b}} m_{v} d(v, b) \\
\quad+2 \sum_{k \in V(C) \backslash\{i, j\}} m_{k}\left(d(j, b)+\frac{m_{i j} m_{j k}}{l}\right) \\
+d(a, i)^{2}+d(j, b)^{2}+2(l+d(a, i)) d(j, b)+\frac{l+2 d(a, i)}{l} d(i, j)(l-d(i, j)) .
\end{gathered}
$$


If $a, b \in V\left(T_{i}\right)$ for some $i$, then it is not difficult to show that

$$
H(a, b)=d(a, b)^{2}+2 \sum_{v \in V(P)} m_{v} d(v, b)
$$

\section{References}

[1] R. Aleliunas, R. M. Karp, R. J. Lipton, L. Lovász, C. W. Rackoff, Random walks, universal travelling sequences, and the complexity of maze problems, in: Proc. 20th Ann. Symp. on Foundations of Computer Science (1979), 218-223.

[2] G. Brightwell and P. Winkler, Maximum hitting time for random walks on graphs, Random Structures Algorithms 1 (1990), 263-276.

[3] A. K. Chandra, P. Raghavan, W. L. Ruzzo, R. Smolensky, and P. Tiwari, The electrical resistance of a graph captures its commute and cover times, Proc. 21st ACM Symp. Theory of Computing (1989), $574-586$.

[4] X. Chang and H. Xu, Chung-Yau invariants and graphs with symmetric hitting times, preprint.

[5] H.Y. Chen, F.J. Zhang, The expected hitting times for graphs with cutpoints, Statist. Probab. Lett. 66 (2004), 9-17.

[6] Fan R. K. Chung, PageRank as a discrete Green's function, Geometry and analysis. No. 1, 285-302, Adv. Lect. Math. (ALM), 17, Int. Press, Somerville, MA, 2011.

[7] Fan R. K. Chung and S.-T. Yau, Discrete green's functions, J. Combin. Theory Ser. A 91 (2000), 191-214.

[8] R. Cogill and C. Peng, A spanning tree method for bounding hitting times of random walks on graphs, SIAM J. Discrete Math. 24 (2010), 808-820.

[9] U. Feige, Collecting Coupons on Trees, and the Analysis of Random Walks, Comput. Complexity 6 (1996/97), no. 4, 341-356. 
[10] A. Georgakopoulos, On walk-regular graphs and graphs with symmetric hitting times, arXiv:1211.5689.

[11] A. Georgakopoulos and S. Wagner, Hitting times, Cover cost, and the Wiener index of a tree, arXiv:1302.3212.

[12] G. Lawler, Expected hitting times for a random walk on a connected graph, Discrete Math. 61 (1986), 85-92.

[13] L. Lovász, Random walks on graphs: A survey, in Combinatorics, Paul Erdös Is Eighty, Bolyai Soc. Math. Stud. 2 (1993), 353-397.

[14] J. Palacios, Bounds on expected hitting times for a random walk on a connected graph, Linear Algebra Appl. 141 (1990), 241-252.

[15] P. Tetali, Random walks and the effective resistance of networks, J. Theoret. Probab. 4 (1991), 101-109.

[16] H. Xu and S.-T. Yau, Discrete Green's functions and random walks on graphs, J. Combin. Theory Ser. A, 120 (2013), 483-499.

Hao Xu

Center of Mathematical Sciences, Zhejiang University, Hangzhou, Zhejiang 310027, China;

Department of Mathematics, University of Pittsburgh, 301 Thackeray Hall, Pittsburgh, PA 15260, USA

E-mail: mathxuhao@gmail.com

Shing-Tung Yau

Department of Mathematics, Harvard University, Cambridge, MA 02138, USA

E-mail: yau@math.harvard.edu 
